\title{
Nicotine replacement therapies: patient safety and persistence
}

\author{
This article was published in the following Dove Press journal: \\ Patient Related Outcome Measures \\ I3 June 201 I \\ Number of times this article has been viewed
}

\author{
Stuart G Ferguson ${ }^{1,2}$ \\ Saul Shiffman ${ }^{3,4}$ \\ Joseph G Gitchell ${ }^{5}$ \\ 'School of Pharmacy, ${ }^{2}$ Menzies \\ Research Institute Tasmania, University \\ of Tasmania, Hobart, Australia; ${ }^{3}$ Pinney \\ Associates, ${ }^{4}$ University of Pittsburgh, \\ Pittsburgh, PA, USA; ${ }^{5}$ Pinney \\ Associates, Bethesda, MD, USA
}

\begin{abstract}
Nicotine replacement therapy (NRT) has become a central part of the treatment of nicotine dependence. However, NRT's potential efficacy is limited to some extent by patient adherence and persistence. Here we review the relationship between NRT compliance and adherence, and overall treatment outcome. We then examine the factors that likely impact on treatment compliance and persistence, with a special focus on users' perceptions of treatment safety and efficacy as possible mediators. Potential clinical strategies for improving suboptimal medication use are also discussed.
\end{abstract}

Keywords: nicotine replacement therapy, compliance, safety

\section{Introduction}

Nicotine replacement products have been marketed for over a quarter of a century, and were available for much of this time over the counter (ie, without a prescription) in many countries. Each year millions of smokers worldwide use a nicotine replacement product as part of a cessation attempt. ${ }^{1,2}$ Since their introduction to the marketplace to aid smoking cessation, the various forms of nicotine replacement therapy (NRT) have become mainstays of the smoking cessation arsenal. NRT is available in multiple forms - chewing gum, lozenges, patch, inhalator, and nasal spray - all of which have been found to be efficacious in aiding smoking cessation. Safety, in particular the adverse event profile of these medications, will be discussed in detail. After this introduction to NRT efficacy and safety, we will turn our attention to issues relating to persistence and compliance, the core focus of this review. In particular, we will discuss the use of NRT during quit attempts (both in the context of controlled clinical trials and in real-world studies), and how this use compares with recommended usage patterns (in terms of daily dosing and duration of use). As we will argue, compliance and persistence are important issues because they directly impact treatment efficacy. Factors that have been found to relate to compliance and persistence, and potential clinical strategies for addressing these deficiencies, are also discussed.

\section{NRT: forms, regimes, and treatment efficacy}

There are currently five forms of NRT approved for use as smoking cessation aids patch, gum, lozenges/oral tablets, inhaler, and nasal spray. These forms were originally developed to be used in isolation (ie, as monotherapies), but, as will be discussed, increasingly researchers are exploring the safety and efficacy of combining NRT products; when used in this fashion, the once-a-day dosing of patch is combined with
School of Pharmacy, University of

Tasmania, Private Bag 26, Hobart,

Tasmania 700I, Australia

Tel +6I 362268536

Fax +61362267627

Email stuart.ferguson@utas.edu.au 
a product that has a more rapid and flexible dosing strategy (such as, gum or lozenges). ${ }^{3}$ The five forms are commonly divided into two groups based on dosing schedule: patch (the only NRT that is designed for once-a-day dosing [steadystate form]) and all other forms (which are designed to be administered at multiple times over the course of the day [ad lib forms]). This division (steady-state versus ad lib) is useful for the purposes of this review. First, the pharmacokinetic profile of the two groups of NRT products are very different, ${ }^{4}$ resulting in different profiles of adverse events from treatment use. Secondly, because the dosing flexibility of ad lib (versus steady-state) NRT products both affords opportunities for strategic use of the products to combat acute cravings ${ }^{5}$ and results in differential susceptibility to compliance issues, the potential interventions for any compliance issues are likely to also differ.

NRT was developed to mitigate the loss of nicotine caused by cessation, thereby reducing craving and withdrawal symptom severity and hence promoting abstinence. ${ }^{6}$ The ability of NRT to reduce craving and withdrawal symptom severity is well documented. ${ }^{5,7}$ It also promotes cessation. Along with varenicline and bupropion, all five forms of NRT are classified as first-line treatments for smoking, ${ }^{8}$ approximately doubling a smoker's odds of achieving long-term abstinence from smoking. ${ }^{3,8,9}$ Table 1 shows the estimated effect of various NRT treatment forms and regimes. For each medication we have expressed the effect of treatment as a relative risk (RR); the likelihood, or probability, of achieving abstinence when using the medication compared to the probability of doing so when treated with a control, or placebo, treatment. A $\mu$ RR value $>1$ indicates participants treated with the target medication are more likely to achieve abstinence than those using the comparison treatment.

While all forms have been found to be efficacious when used as monotherapies (Table 1), the projected treatment outcomes show variation across forms, with projected 6-month abstinence rates ranging from $27 \%$ (for nicotine nasal spray) to $19 \%$ (for nicotine gum) (with a projected base rate for placebo quitting of $14 \%) .{ }^{8}$ However, both direct and indirect comparisons have failed to show a significant difference across the various forms of NRT monotherapy, suggesting that all forms of NRT are equally efficacious. ${ }^{3}$

Combination NRT - that is, utilizing multiple forms of NRT during a quit attempt - appears to be more efficacious that using a single NRT (Table 1)..$^{3,8,10,11}$ Note that the RR shown in Table $1-1.35$ - is calculated using standard patch treatment as the comparison group (rather than a placebo treatment). The mechanism through which this boost in
Table I The effect of various forms and regimes of NRT on treatment outcome

\begin{tabular}{|c|c|}
\hline NRT form & $\operatorname{RR}(95 \% \mathrm{Cl})$ \\
\hline Overall ${ }^{\mathrm{a}}$ & $1.58(1.50-1.66)$ \\
\hline \multicolumn{2}{|l|}{ Monotherapies } \\
\hline Patch & $1.66(1.53-1.81)$ \\
\hline Standard gum & $1.43(1.33-1.53)$ \\
\hline Lozenges $^{b}$ & $2.00(1.63-2.43)$ \\
\hline Inhaler & $1.90(1.36-2.67)$ \\
\hline Spray & $2.02(1.49-3.73)$ \\
\hline \multicolumn{2}{|l|}{ Incremental treatments } \\
\hline Pre-quit gum ${ }^{c}$ & $0.87(0.60-1.26)$ \\
\hline Pre-quit patch ${ }^{d}$ & $2.17(1.46-3.22)$ \\
\hline \multicolumn{2}{|l|}{ Combination therapy } \\
\hline Patch + ad lib NRT (gum/spray/inhaler) ${ }^{d}$ & $1.35(1.11-1.63)$ \\
\hline
\end{tabular}

Notes: a Meta-analysis using outcomes from all monotherapy forms of NRT; ' Includes studies of oral nicotine tablets; ${ }^{3}$ RR calculated using standard gum treatment as reference; ${ }^{d R R}$ calculated using standard patch treatment as reference; RR sources: Lindson and Aveyard; ${ }^{17}$ Shiffman and Ferguson; ${ }^{16}$ Stead at al. ${ }^{3}$

Abbreviations: NRT, nicotine replacement therapy; RR, relative risk; $\mathrm{Cl}$, confidence interval.

efficacy is achieved is as yet unknown. The effect is unlikely to be driven simply by higher nicotine dosing: higher-dose monotherapy patch treatment ( $>25 \mathrm{mg} / 24$ hours), for example, does not appear to improve efficacy beyond standard maximum patch doses. ${ }^{8}$ It may be that the combination of an ad lib NRT (such as gum or lozenges) on top of the steady-state patch treatment helps smokers to resist cueinduced cravings, which are theorized to be important in promoting relapse. ${ }^{5}$ While monotherapy patch successfully attenuates background, or tonic, craving, ${ }^{12}$ it does not appear to block cue-induced cravings ${ }^{13}$ or aid in recovery from such cravings, ${ }^{14}$ whereas nicotine gum and lozenges do appear to provide this latter benefit. ${ }^{9,15}$

Researchers have also started to explore the efficacy of other ways of using NRT. Reducing the salience of smoking cues has also been suggested as a mechanism for explaining the improvement in efficacy achieved through pre-quit patch use, another novel usage of existing NRT (Table 1). The noncontingent delivery of nicotine achieved via patch may help to break some of the environmental cues associated with smoking when delivered during ad lib smoking. ${ }^{16}$ If this mechanism of action explains the improvement in efficacy achieved by using nicotine patch prior to quitting, it would help to explain the seemingly contradictory finding that prequit gum use does not appear to result in similar improvements in treatment outcome (Table 1). ${ }^{17}$ It is likely that, when used before quitting, gum is used instead of smoking - that is, as a replacement to individual cigarettes - a regime than is unlikely to break the association between cues and smoking compared with noncontingent nicotine administration in 
the form of patch. Regardless of the mechanism, these two alternate NRT regimes - combination NRT use and pre-quit patch use - show substantial promise as smoking cessation treatments; further research will be needed to confirm the true magnitude of their benefit and the mechanisms of action. ${ }^{17}$ Finally, it should also be noted that nicotine patch has also been tested in combination with other smoking cessation aids (eg, bupropion sustained release, varenicline, nortriptyline) and with other nonapproved agents (eg, paroxetine, mecamylamine), with limited success (results not reported here). ${ }^{8}$

\section{NRT safety}

The duration of time that NRT has been studied - both experimentally and under real-world actual-use conditions has given researchers and clinicians ample time to study the adverse events and side effects of NRT use. Results from scores of clinical trials suggest that serious adverse events associated with NRT are rare and typically cease once treatment has been discontinued. ${ }^{8}$ This is arguably not particularly surprising, given that the active ingredient of NRT - nicotine - is an agent that smokers have demonstrated that they can safely tolerate.

Because of differences in the speed of nicotine delivery and the mode of delivery, side effects of NRT use vary across forms. The most commonly reported side effect across NRT forms is nausea but even this is relatively rare: a metaanalysis of studies involving nicotine patch, for example, found that nausea was reported by only $5 \%$ of patients. ${ }^{18}$ A quarter $(25 \%)^{18}$ of patch users report localized skin irritation due to the patch itself (eg, from the adhesives used to hold the patch to the skin), but these tend to be mild and can be ameliorated by rotating the patch application site and/or through the application of topical creams (eg, hydrocortisone or triamcinolone). ${ }^{8}$ Some users $(19 \%)^{18}$ report sleep disturbance when using patch; sufferers can be advised to either remove the 24-hour patch when going to bed at night, or change to a 16 -hour patch if this symptom persists. ${ }^{8}$

Unique side effects for the three oral forms of NRT gum, lozenges, and inhaler - also result from the method of delivery. Gum users may experience problems resulting from chewing gum, such as mouth soreness, hiccups, dyspepsia, and jaw ache. ${ }^{8}$ Similarly, nicotine lozenge use is associated with hiccups and heartburn, both likely due to the oral delivery system. Some of these side effects may be related to the way these products are being used (eg, the chewing technique utilized with gum); guidance on appropriate product use can help to reduce their occurrence. Almost half of nicotine inhaler users report experiencing local irritation in the mouth and throat following use. As with other side effects of NRT use, these side effects are generally mild and transient, ceasing as soon as NRT use is discontinued. Finally, nicotine nasal spray users typically report some degree of nasal irritation: over $90 \%$ of users report experiencing such symptoms within the first days of use, ${ }^{8}$ with the majority of these still experiencing mild to moderate irritation for up to a month. Other side effects related to the delivery mechanism include nasal congestion and changes in sense of smell and taste.

Moving on from the products themselves, nicotine itself has many effects on the body. Perhaps most notably in terms of potential side effects of NRT use, nicotine administration has been shown to directly affect the cardiovascular system, increasing both heart rate and blood pressure. ${ }^{19,20}$ For this reason, NRT products - and in particular patch - have historically carried warnings regarding use in patients who have a history of cardiovascular problems, such as myocardial infarction (heart attack), arrhythmias, or unstable/uncontrolled angina pectoris. ${ }^{21}$ However, fears of NRT exacerbating such conditions appear to be unfounded. Controlled studies have demonstrated that, even in groups of smokers at high risk, the use of nicotine replacement products does not appear to significantly increase the likelihood of cardiovascular events, ${ }^{22}$ and NRT has been found to be safe for use with hypertensive patients. ${ }^{21,23,24}$

One relatively recent study purports to demonstrate a link between pure nicotine and tumor growth, opening up the possibility that NRT may promote cancer growth in users. Heeschen and colleagues ${ }^{25}$ in vitro findings show that pure nicotine may stimulate angiogenesis (the growth of new blood vessels), a necessary step in tumor growth. Again, however, the clinical evidence fails to support this finding: there is no clinical evidence that either pure nicotine or NRT cause cancer in humans. ${ }^{20,26}$ In sum, when compared to the accepted dangers of continued smoking, nicotine delivered via NRT products appears to carry negligible health risks.

The final safety concerns with NRT use that we will discuss here are the dependence potential of these medications, and, relatedly, the potential for withdrawal symptoms to manifest after stopping NRT use. Nicotine is well-known as being a drug of dependence, so it is theoretically possible for users to become dependent on medications that contain this agent, and for them to display signs of withdrawal upon cessation of use. An important difference between NRT and cigarettes in this regard, however, is the speed at which peak blood levels of nicotine are achieved. The dose of nicotine delivered by the currently approved nicotine medications, and the speed 
at which that nicotine is delivered, are substantially lower than those achieved by cigarettes. ${ }^{27}$ (Indeed, this fact has led some to argue that the approved NRT doses are too low and effectively underdose some smokers. ${ }^{28}$ ) Consequently, the theoretical dependence potential of these medications is considered low. Surveillance data from NRT sales data support this conclusion, suggesting that the vast majority of users do not maintain NRT use beyond the recommended therapeutic treatment duration. ${ }^{29}$ The one possible exception to this is nicotine spray: of the available medications, nicotine nasal spray produces higher peak nicotine levels ${ }^{27}$ and is believed to have the highest dependence potential; ${ }^{8}$ and some studies suggest that $15 \%-20 \%$ of users continue use for longer than the recommended duration. ${ }^{8}$ However, it is unclear whether such extended use is truly a sign of dependence, or rather a continued attempt to remain smoke free. ${ }^{21}$ With regard to withdrawal symptoms, there is little research in this area that we know of, but the data that exist ${ }^{30}$ suggest that there is no pronounced or significant withdrawal syndrome from cessation of NRT.

In summary, the NRT products have all been well documented to be both safe and effective. Side effects are rare and generally mild and transient. These products appear to be well tolerated. Finally, despite initial concerns due to their active ingredient, NRT products do not appear to carry a high risk of dependence/extended nontherapeutic use; indeed as we will discuss in the following section, if anything most forms of NRT suffer from the opposite extreme, with users not administering enough doses, or maintaining use for long enough.

\section{Adherence and compliance: observed NRT use and impact on treatment outcome}

Like perhaps all other multidose medications, compliance with smoking cessation medications is suboptimal. Numerous studies have found that smokers consistently underutilize NRT, both in terms of the number of pieces administered per day (commonly referred to as dosing "compliance") and the duration of time that treatment is used (which we will refer to here as treatment "persistence"). Across forms, smokers have been found to consistently underdose: using fewer doses/patches per day (in the case of ad lib NRT forms in particular) $)^{9,31}$ and ceasing treatment prior to the end of the recommended treatment course. ${ }^{31}$ In one large national survey, ${ }^{31}$ for example, less than half of recent users reported that they had used gum or patch for greater than 4 weeks; and less than a quarter reported using the recommended nine-plus pieces of nicotine gum per day during treatment.

Issues with dosing compliance are of interest to researchers and clinicians primarily because they stunt the potential efficacy of these medications. Like all drugs, NRT's efficacy is linked to compliance-smokers must actually use sufficient quantities of the medication, and over a sufficient duration of time, for it to be effective in aiding smoking cessation. The relationship between dosing compliance and treatment outcome has been studied most comprehensively in the ad lib forms of NRT, in particular with nicotine gum and lozenges. Studies have consistently found a positive relationship between the number of pieces of gum/lozenges, or the number of sprays, used per day and treatment outcome, with smokers who use a greater number of doses per day having a greater likelihood of achieving abstinence. ${ }^{32-34}$ A similar relationship has been reported with nicotine patch. ${ }^{35}$

This relationship between compliance and treatment outcome appears to be driven pharmacologically - that is, compliance use results in sufficient amounts of medicine being delivered. Because the observed relationship between use and outcome is correlational, it is difficult - not to mention ethically questionable - to randomly assign patients to compliant versus noncomplaint use. One might be tempted to conclude that the relationship between compliance and treatment outcome is noncausal. Shiffman articulated three alternate explanations for the observed relationship between treatment outcome and medication compliance: (1) that a third confounding variable affects both medication use and treatment outcome; (2) that the behaviors associated with medication use, regardless of its nicotine content, might affect treatment outcome; or (3) that the observed relationship between compliance and outcome is causal, but that relapse to smoking actually drives noncompliance, rather than the other way around (reverse causation). ${ }^{33}$ Shiffman went on to present a series of analyses that tested each of these alternate explanations and concluded that they were unsupported: the improved treatment outcomes seen among compliance users appear to be due to increased use of these efficacious medications. ${ }^{33}$

The relationship between the length of NRT use and treatment outcome is less clear. NRT labeling typically advises users to remain on treatment for 10 to 12 weeks, but some regulatory jurisdictions allow use to continue for up to 6 months as a relapse prevention strategy. (At the far extreme, in 2010, the UK's Medicines and Healthcare Products Regulatory Agency completely eliminated the duration of use limit for NRT). While a number of studies have examined 
the effectiveness of longer-term relapse prevention use of NRT compared with the standard 10 to 12 -week course (with mixed results), ${ }^{3,8}$ few have closely examined the relationship between persistence and outcome within the standard duration of treatment. Given the pharmacologically driven relationship between compliance and treatment, however, it would seem plausible that treatment persistence would also promote abstinence.

Given that compliant use is linked to treatment outcome, we will now turn our attention to studies that have attempted to understand noncompliant usage patterns, and to influence more optimal medication use. Sadly, as will be discussed, the outcomes of such endeavors to date have been frustratingly poor.

\section{Drivers of noncompliant use and potential interventions}

To improve compliance with NRT dosing, it is first necessary to understand the drivers, or root causes, of the noncompliant behavior; without knowing why a patient is noncompliant with dosing instructions it is impossible to know how to design and implement a successful intervention. Unfortunately, while there are numerous potential reasons for poor compliance and adherence to NRT, we know of few published studies that have attempted to identify self-reported reasons for these usage patterns. A recent study of NRT users found that, of those who had discontinued use prior to the end of the recommended regime for a reason other than having relapsed, the most commonly reported reason for prematurely ceasing treatment was because it was felt that NRT was no longer necessary ( $\sim 30 \%$ of users); side effects $(\sim 28 \%)$ and practical reasons (eg, cost, ran out of the NRT) were also frequently cited. ${ }^{36}$ The organoleptic characteristics of the products themselves (eg, taste of gum/lozenges, mouth and throat irritation from the spray) may also influence compliance; this suggests that ongoing development of NRT delivery products may prompt improved compliance (by increasing the likelihood that users can find a nicotine replacement product that they can tolerate using).

Paradoxically, the stated belief that NRT is no longer needed as part of a quit attempt may actually stem from the efficacy of NRT: when craving and withdrawal are well controlled via treatment, patients may mistakenly assume that the treatment itself is actually unnecessary. As such, this may highlight the need to educate users on the role of NRT in quitting. The exact role and effects of treatment may not be the only aspect of medication use that smokers are misinformed about: a number of studies have reported that a large proportions of smokers have concerns about the safety and efficacy of NRT products, believing either that these products do not promote cessation, that they are dangerous/ harmful to use, or that the mechanism of action of nicotine replacement products is to make a user sick if they lapse during treatment. ${ }^{31,37,38}$ Important for the purposes of this discussion, smokers who hold these misperceptions are less likely to have used NRT in the past, and less likely to say that they intend to use it as part of future quit attempts, ${ }^{31,37,38}$ suggesting that beliefs about NRTs' safety and efficacy may impact on treatment use. Further, one of these studies also reported that safety and efficacy concerns were related to poorer compliance and adherence: gum users with concerns about the safety of NRT reported using fewer pieces of gum per day during treatment, and were less likely to report that they used the gum for more than 4 weeks. ${ }^{31}$

Such findings suggest that providing education and advice to patients regarding the safety and efficacy of NRT may promote compliant use. One study, however, that tried such an intervention with interested quitters was unsuccessful - providing brief education to gum users did not significantly change either the number of pieces of gum used per day, or the percentage of users who were categorized as compliant users. ${ }^{39}$ A more recent study in a population of smokers interested in reduction also found that providing education on the importance of compliance did not improve subsequent compliance with NRT dosing. ${ }^{40}$ It is possible that the interventions themselves in these studies were not targeted enough to be effective; multicomponent strategies may be required to successfully change peoples' perceptions of safety and efficacy. If such programs were developed, and were found to be effective, implementation on a population level would nevertheless be challenging. More speculatively, a purely behavioral strategy of routine use may help to overcome misperceptions by automating the process of use and, hence, simply circumventing the higher-level cognitive processes involved in weighing up the risk-benefit of additional use. Such a behavioral approach would also address a more parsimonious explanation of poor treatment compliance - perhaps users do not comply simply because they forget to take the medication at the allotted times. Of course, it is also possible that safety and efficacy concerns are simply not as important in determining compliant use as the correlational data suggest. More research is needed to determine whether safety and efficacy misperceptions are drivers of noncompliant use, and, if so, whether these views can be changed and subsequently user improved. 
Moving on, as noted earlier, a survey of NRT users found that motivational factors such as the cost of NRT were often cited as a reason for early cessation of treatment. ${ }^{36}$ This concurs with research on barriers to NRT use: in one survey, $53 \%$ of smokers who previously tried to quit but who had not used NRT cited the cost of these medications as a reason. ${ }^{41}$ This would suggest that providing NRT for free would boost compliance and adherence. In a recent nicotine gum study, Mooney and colleagues ${ }^{39}$ tested a related strategy: not only was treatment provided free of charge, but also participants were offered additional financial rewards for complying with dosing instructions. Over a 15-day intervention period, patients in the contingency management group of Mooney and colleagues' ${ }^{39}$ study were told that they would be paid for each day that they reported chewing at least 12 pieces of gum per day. The intervention was somewhat successful: patients in the contingency management group reported using approximately four pieces of gum per day more than patients in a usual care group, and two thirds (66\%) of these patients were categorized as compliant gum users during the study period (as opposed to just 14\% of the usual care participants). However, the fact that compliance and adherence is generally poor even in clinical trials of NRT, where drug treatment is typically provided free of charge, would seem to contradict the notion that cost is a primary barrier to NRT compliance and adherence. Furthermore, providing education to smokers about the safety and efficacy of NRT increases their interest in using NRT as part of a future quit attempt, even among those who cited cost as a reason for not having used it in the past; suggesting that cost is not the sole barrier to NRT uptake. ${ }^{41}$ Thus, in summary, while the cost of NRT may negatively impact on compliance and adherence to some extent, evidence suggests that cost is unlikely to be the root cause of noncompliance.

\section{Conclusion}

Medicinal nicotine in all of its currently available forms has been comprehensively demonstrated to be effective as an aid for smoking cessation. Such products have also been found to be safe, with adverse events typically mild and transient, ceasing when treatment is stopped. Sadly, despite these characteristics, compliance and persistence with NRT are typically poor and such suboptimal deviations from the recommended usage patterns negatively impact on treatment outcome. As discussed, many factors likely affect treatment compliance and persistence. In this review we focused on users' perceptions of treatment safety and efficacy as possible mediators and discussed preliminary potential clinical strategies for addressing these deficiencies. Sadly, the literature is currently silent on effective strategies for improving compliance. Any successful intervention is likely to require multiple components and focus on multiple drivers of suboptimal medication use.

\section{Disclosure}

GlaxoSmithKline Consumer Healthcare provided financial support to Dr Ferguson, Mr Gitchell, and Dr Shiffman for the preparation of this manuscript, but had no role in its drafting. Through their work at Pinney Associates, Dr Shiffman and Mr Gitchell serve as consultants to GlaxoSmithKline Consumer Healthcare on an exclusive basis on matters relating to smoking cessation. Dr Shiffman and $\mathrm{Mr}$ Gitchell also have an interest in a venture to develop a new nicotine replacement medication.

\section{References}

1. Lader D, Goddard E. Smoking Related Behaviour and Attitudes: 2003. London: Office for National Statistics; 2004.

2. Shiffman S, Brockwell SE, Pillitteri JL, Gitchell JG. Use of smokingcessation treatments in the United States. Am J Prev Med. 2008; 34(2):102-111.

3. Stead LF, Perera R, Bullen C, Mant D, Lancaster T. Nicotine replacement therapy for smoking cessation. Cochrane Review. 2008;1:CD000146. Doi:10.1002/14651858.CD000146.pub3.

4. Fant RV, Owen LL, Henningfield JE. Nicotine replacement therapy. Prim Care. 1999;26(3):633-652.

5. Ferguson SG, Shiffman S. The relevance and treatment of cueinduced cravings in tobacco dependence. J Subst Abuse Treat. 2009; 36(3):235-243.

6. Jarvik ME, Henningfield JE. Pharmacological treatment of tobacco dependence. Pharmacol Biochem Behav. 1988;30(1):279-294.

7. Rose JE, Levin ED, Behm FM, Adivi C, Schur C. Transdermal nicotine facilitates smoking cessation. Clin Pharmacol Ther. 1990;47(3): 323-330.

8. Fiore MC, Jaen CR, Baker TB, et al. Treating tobacco use and dependence: 2008 update; clinical practice guideline. Rockville, MD: United States Department of Health and Human Services; 2008.

9. Shiffman S, Dresler CM, Hajek P, Gilburt SJ, Targett DA, Strahs KR. Efficacy of a nicotine lozenge for smoking cessation. Arch Intern Med. 2002;162(11):1267-1276.

10. Piper ME, Smith SS, Schlam TR, et al. A randomized placebo-controlled clinical trial of 5 smoking cessation pharmacotherapies. Arch Gen Psychiatry. 2009;66(11):1253-1262.

11. Smith SS, McCarthy DE, Japuntich SJ, et al. Comparative effectiveness of 5 smoking cessation pharmacotherapies in primary care clinics. Arch Intern Med. 2009;169(22):2148-2155.

12. Shiffman S, Ferguson SG. The effect of a nicotine patch on cigarette craving over the course of the day: results from two randomized clinical trials. Curr Med Res Opin. 2008;24(10):2795-2804.

13. Tiffany ST, Cox LS, Elash CA. Effects of transdermal nicotine patches on abstinence-induced and cue-elicited craving in cigarette smokers. J Consult Clin Psychol. 2000;68(2):233-240.

14. Waters AJ, Shiffman S, Sayette MA, Paty JA, Gwaltney CJ, Balabanis MH. Cue-provoked craving and nicotine replacement therapy in smoking cessation. J Consult Clin Psychol. 2004;72(6):1136-1143.

15. Shiffman S, Shadel WG, Niaura R, et al. Efficacy of acute administration of nicotine gum in relief of cue-provoked cigarette craving. Psychopharmacology (Berl). 2003;166(4):343-350. 
16. Shiffman S, Ferguson SG. Nicotine patch therapy prior to quitting smoking: a meta-analysis. Addiction. 2008;103(4):557-563.

17. Lindson N, Aveyard P. Pre-loading an updated meta-analysis of nicotine preloading for smoking cessation: investigating mediators of the effect. Psychopharmacology (Berl). Nov 2010. [Epub ahead of print].

18. Greenland S, Satterfield MH, Lanes SF. A meta-analysis to assess the incidence of adverse effects associated with the transdermal nicotine patch. Drug Saf. 1998;18(4):297-308.

19. Benowitz NL. Pharmacology of nicotine: addiction, smoking-induced disease, and therapeutics. Annu Rev Pharmacol Toxicol. 2009;49: $57-71$.

20. Royal College of Physicians. Harm reduction in nicotine addiction: helping people who can't quit. A report by the Tobacco Advisory Group of the Royal College of Physicians. London: Royal College of Physicians; 2007.

21. Zapawa LM, Hughes JR, Benowitz NL, Rigotti NA, Shiffman S. Cautions and warnings on the US OTC label for nicotine replacement: what's a doctor to do? Addict Behav. 2011;36(4):327-332.

22. Joseph AM, Norman SM, Ferry LH, et al. The safety of transdermal nicotine as an aid to smoking cessation in patients with cardiac disease. N Engl J Med. 1996;335(24):1792-1798. Erratum in: N Engl J Med. 2007;356(24):2554. Antonucio, DO.

23. Benowitz NL. Safety of nicotine in smokers with hypertension. Am J Hypertens. 2001;14(7 Pt 1):731-732.

24. Hubbard R, Lewis S, Smith C, et al. Use of nicotine replacement therapy and the risk of acute myocardial infarction, stroke, and death. Tob Control. 2005;14(6):416-421.

25. Heeschen C, Jang JJ, Weis M, et al. Nicotine stimulates angiogenesis and promotes tumor growth and atherosclerosis. Nat Med. 2001;7(7) 833-839.

26. Murray RP, Connett JE, Zapawa LM. Does nicotine replacement therapy cause cancer? Evidence from the Lung Health Study. Nicotine Tob Res. 2009;11(9):1076-1082.

27. Sweeney CT, Fant RV, Fagerstrom KO, McGovern JF, Henningfield JE Combination nicotine replacement therapy for smoking cessation: rationale, efficacy and tolerability. CNS Drugs. 2011;15(6):453-467.

28. Dale LC, Hurt RD, Offord KP, Lawson GM, Croghan IT, Schroeder DR. High-dose nicotine patch therapy: percentage of replacement and smoking cessation. JAMA. 1995;274(17):1353-1358.

29. Shiffman S, Hughes JR, Pillitteri JL, Burton SL. Persistent use of nicotine replacement therapy: an analysis of actual purchase patterns in a population based sample. Tob Control. 2003;12(3):310-316.
30. Hurt RD, Offord KP, Lauger GG, et al. Cessation of long-term nicotine gum use: a prospective, randomized trial. Addiction. 1995;90(3): 407-413.

31. Shiffman S, Ferguson SG, Rohay J, Gitchell JG. Perceived safety and efficacy of nicotine replacement therapies among US smokers and ex-smokers: relationship with use and compliance. Addiction. 2008; 103(8):1371-1378.

32. Jackson PH, Stapleton JA, Russell MA, Merriman RJ. Nicotine gum use and outcome in a general practitioner intervention against smoking. Addict Behav. 1989;14(3):335-341.

33. Shiffman S. Use of more nicotine lozenges leads to better success in quitting smoking. Addiction. 2007;102(5):809-814.

34. Tonnesen P, Norregaard J, Mikkelsen K, Jorgensen S, Nilsson F. A double-blind trial of a nicotine inhaler for smoking cessation. JAMA 1993;269(10):1268-1271.

35. Shiffman S, Sweeney CT, Ferguson SG, Sembower MA, Gitchell JG. Relationship between adherence to daily nicotine patch use and treatment efficacy: secondary analysis of a 10-week randomized, doubleblind, placebo-controlled clinical trial simulating over-the-counter use in adult smokers. Clin Ther. 2008;30(10):1852-1858.

36. Balmford J, Borland R, Hammond D, Cummings KM. Adherence to and reasons for premature discontinuation from stop-smoking medications: data from the ITC four-country survey. Nicotine Tob Res. 2011;13(2):94-102.

37. Bansal MA, Cummings KM, Hyland A, Giovino GA. Stop-smoking medications: who uses them, who misuses them, and who is misinformed about them? Nicotine Tob Res. 2004;6 Suppl 3:S303-S310.

38. Vogt F, Hall S, Marteau TM. Understanding why smokers do not want to use nicotine dependence medications to stop smoking: qualitative and quantitative studies. Nicotine Tob Res. 2008;10(8):1405-1413.

39. Mooney ME, Babb D, Jensen J, Hatsukami DK. Interventions to increase use of nicotine gum: a randomized, controlled, single-blind trial. Nicotine Tob Res. 2005;7(4):565-579.

40. Chan SSC, Leung DYP, Abdullah ASM, Wong VT, Hedley AJ, Lam T. A randomized controlled trial of a smoking reduction plus nicotine replacement therapy intervention for smokers not willing to quit smoking. Addiction. Jan 13 2011. [Epub ahead of print].

41. Ferguson SG, Gitchell JG, Shiffman S, Sembower MA, Rohay JM, Allen J. Providing accurate safety information may increase a smoker's willingness to use nicotine replacement therapy as part of a quit attempt. Addict Behav. Feb 13 2011. [Epub ahead of print].
Patient Related Outcome Measures

\section{Publish your work in this journal}

Patient Related Outcome Measures is an international, peer-reviewed, open access journal focusing on treatment outcomes specifically relevant to patients. All aspects of patient care are addressed within the journal and practitioners from all disciplines are invited to submit their work as well as healthcare researchers and patient support groups. Areas covered will

\section{Dovepress}

include: Quality of life scores; Patient satisfaction audits; Treatment outcomes that focus on the patient; Research into improving patient outcomes; Hypotheses of interventions to improve outcomes; Short communications that illustrate improved outcomes; Case reports or series that show an improved patient experience; Patient journey descriptions or research. 